\title{
Optimal Design of Camshaft's Processing Technology
}

\author{
Dongfang $\mathrm{Hu}^{1}$ and Jianwei $\mathrm{Guo}^{2}$ \\ ${ }^{1}$ School of Mechanical and Electrical Engineering; \\ ${ }^{1}$ Henan University of Science and Technology, Luoyang, Henan 471003
}

\begin{abstract}
In order to achieve optimization of camshaft NC turning method, this paper has analyzed processing method of camshaft in traditional processing technology and proposed Project of merging turning process and shortening processing chain of camshaft. What's more, it has also analyzed process scheme, tool angle selection and tool path mode of turning. At the same time, it has carried out numerical analysis on cutting parameter choice by optimal tool of Matlab software. It has operated machining of camshaft on reformed $\mathrm{CNC}$ lathe and verified cutting process optimization effectiveness of camshaft NC cutting.
\end{abstract}

Keywords: Camshaft; NC turning; Processing chain; Cutting parameter; Machining

\section{凸轮轴的加工工艺优化设计 \\ 胡东方 ${ }^{1}$ ，郭建伟 ${ }^{1}$ \\ (1 河南科技大学机电工程学院, 河南 洛阳 471003)}

摘要: 为实现凸轮轴数控车削方法的优化, 分析了凸轮轴在传统加工工艺的加工方法, 提出了合并车削工艺, 缩短凸轮 轴加工工艺链的方案, 并对车削的工艺方案、刀具角度选择、走刀方式等进行了分析, 同时用 Matlab 软件的优化工具对切 削用量的选择进行了数值分析。通过在改造后的数控车床上进行了凸轮轴的切削加工, 验证了凸轮轴数控切削工艺优化的有 效性。

关键词: 凸轮轴；数控车削；工艺链；切削用量；切削加工

中图分类号: TH 132.47 文献标志码: A

引言

凸轮轴作为发动机配气机构的控制部件, 其加工精度对燃油效率起决定性作用, 凸轮型面质量的高低 直接影响着动力的发挥 ${ }^{[1]}$ 。近年来随着发动机性能的提升, 作为关键传动部件之一的凸轮轴也有了较大的 改进, 其加工方式也随着计算机技术的发展而高速发展。2010 年南车戚墅堰机车有限公司的夏焕金、陆忠 民开展了凸轮轴型面车铣加工工艺的类似研究。发现无论是轴向车铣还是正交车铣, 其加工精度和效率都 较传统的仿形靠模车削有较大的提高 ${ }^{[2]}$ 。2012 年华中科技大学的郑孟昆等人在进行高精度凸轮轴磨床砂轮 架优化设计的研究中, 提出了建立恒线速凸轮轴磨削的运动学模型, 对于保证凸轮型面质量有重要意义 ${ }^{[3]}$ 。 2012 年, 德国弗劳恩霍夫机床与成型技术研究所的 Neugebauer, R 等人, 提出优化凸轮轴加工过程链的“混 合加工” 方法来提高加工效率，实现高效切削 (HPC)。凸轮轴轴身既细且长，加工过程中，刚性差、易跳 动、易变形弯曲并且有震纹, 磨削精加工时易烧伤, 所以其工艺性较差。现在对发动机性能要求越来越高, 国家到 2018 年要推行 “国五” 排放标准, 要求更高的三低一高（低消耗、低排放、低污染、高环保）。 这就对凸轮轴的加工质量和效率有了更高的要求。

\section{1 凸轮轴加工工艺分析}

凸轮轴的切削工艺主要包括粗加工、精加工和其它一些辅助工艺, 辅助工艺中的调质处理工艺, 目的 
是消除粗加工内应力, 提高机械性能, 同时中心孔是各项工序的定位基准, 精加工前要清洗、修整中心孔。

\section{1 传统凸轮轴加工工艺缺陷分析}

传统加工工序的特点包括: (1)在粗加工阶段中凸轮轴的各支撑轴颈、开档用车削加工, 用大的切削用 量尽快切除大部分金属, 粗加工毛坯后, 留少许精加工余量。大切削用量能尽快发现零件缺陷, 作报废处 理。轴颈、开档部分采用普通车床加工，凸轮部分采用靠模车床加工。一种零件用一种靠模，柔性差，调 整困难。如果凸轮零件品种多, 机床的调整就更不经济。(2)在精加工阶段中凸轮轴零件的精加工含各支撑 轴颈、凸轮外形的磨削加工以及两端面孔的钻铰加工, 两端面孔的加工主要在摇臂钻床上加工, 凸轮轴经 过车钻加工后, 要转至磨床上进行磨削精加工, 传统的凸轮轴磨床也需要精密靠模, 为了保证精度, 尤其 是一些重要的位置精度, 企业需要购置一批专机来制作靠模。这些成本摊薄到每个零件上, 会大幅度提高 零件价格, 降低企业市场竞争力。

根据以上对凸轮轴的零件分析，本文整理了凸轮轴传统加工工艺流程，如表 1 所示

\begin{tabular}{|c|c|c|c|c|}
\hline \multirow{2}{*}{$\begin{array}{l}\text { 序 } \\
\text { 号 }\end{array}$} & \multirow{2}{*}{ 工序名称 } & \multirow{2}{*}{ 机床名称 } & \multicolumn{2}{|c|}{ 单件工时 (分钟) } \\
\hline & & & 机动时间 & 辅助时间 \\
\hline 1 & 铣端面打中心孔 & 铣端面、铣中心孔机组 & 5 & 2 \\
\hline 2 & $\begin{array}{l}\text { 粗车齿轮轴颈及各支撑轴 } \\
\text { 颈 }\end{array}$ & 普通车床 & 8 & 2 \\
\hline 3 & 车开档 & 普通车床 & 5 & 2 \\
\hline 4 & 车末端支撑轴颈 & 液压仿形车床 & 3 & 1 \\
\hline 5 & 车其余支撑轴颈 & 数控车床 & 5 & 2 \\
\hline 6 & 钻铰螺纹孔及销孔 & 摇臂钻 & 5 & 2 \\
\hline 7 & 车凸轮外形 & 凸轮车床 & 8 & 2 \\
\hline 8 & 车油槽 & 液压仿形凸轮车床 & 5 & 2 \\
\hline 9 & 粗磨凸轮外形 & 凸轮磨床 & 10 & 2 \\
\hline 10 & 热处理 & & 批处理 & \\
\hline 11 & 车两端支撑轴颈倒角 & 普通车床 & 3 & 2 \\
\hline 12 & $\begin{array}{l}\text { 修整两端中心孔和车沉割 } \\
\text { 槽 }\end{array}$ & 普通车床 & 8 & 2 \\
\hline 13 & 精磨全部支撑轴颈 & 半自动外圆磨床 & 10 & 2 \\
\hline 14 & $\begin{array}{l}\text { 精磨齿轮安装轴颈及两止 } \\
\text { 推面 }\end{array}$ & 半自动端面外圆磨床 & 10 & 2 \\
\hline 15 & 铣键槽 & 卧式铣床 & 5 & 2 \\
\hline 16 & 精磨全部凸轮外形 & 凸轮轴数控磨床 & 10 & 2 \\
\hline
\end{tabular}

表 1 凸轮轴传统加工工艺流程

\section{2 数控切削凸轮轴型面轮廓的优越性}

零件的加工时间包括机动工时和辅助工时两部分。由表 1 可以看出单件凸轮轴加工的工艺流程相当长, 2、3、4、5、7、8 工序都是放在车床上车削的工序，第 6 道工序钻扩铰螺纹孔及销孔是为第 15 道工序铣 键槽做定位用的，因此，我们不妨把 2、3、4、5、7、8 六道工序放在一台改进后的数控车床上加工，就 能缩减加工时间，尤其是工序之间的 9 分钟辅助工时就可以避免。同时一次装夹，有利于提高位置精度。 同时凸轮轴的数控切削可不必制作靠模, 经验证明, 凸轮轴的加工轮廓误差的 $30 \%$ 是由靠模误差引起的 ${ }^{[4]}$, 因此抛弃靠模, 还有助于提高表面轮廓精度。参考表 2 所示各种加工方式所能达到的精度 ${ }^{[5]}$, 综合考虑质 量和效率两方面的因素，可以先用车削加工凸轮型面和轴颈外圆以提高效率，最后用磨削保证设计质量。 


\begin{tabular}{|c|c|c|c|}
\hline \multicolumn{2}{|c|}{ 加工方法 } & 精度等级 IT & 粗糙度 $R_{a} / \mu \mathrm{m}$ \\
\hline \multirow{3}{*}{ 车削 } & 粗车 & $12^{\sim} 13$ & $12.5^{\sim} 50$ \\
\hline & 细车 & $10^{\sim} 11$ & $1.6 \sim 6.3$ \\
\hline & 精车 & $6^{\sim} 9$ & $0.2^{\sim} 1.6$ \\
\hline \multirow{3}{*}{ 铣削 } & 粗铣 & $11^{\sim} 13$ & $3.2^{\sim} 12.5$ \\
\hline & 细铣 & $10 \sim 11$ & $0.8^{\sim} 3.2$ \\
\hline & 精铣 & $6 \sim 9$ & $0.2^{\sim} 0.8$ \\
\hline \multirow{3}{*}{ 磨削 } & 粗磨 & $7^{\sim} 9$ & $0.8^{\sim} 1.6$ \\
\hline & 细磨 & $6 \sim 8$ & $0.2^{\sim} 0.4$ \\
\hline & 精磨 & $5^{\sim} 7$ & $0.05^{\sim} 0.1$ \\
\hline
\end{tabular}

表 2 加工方式及其精度

凸轮轴型面数控切削工艺把计算机技术、自动化技术及传统切削工艺与设备相结合, 增加了设备的柔 性, 降低了成本, 提高了精度, 易于开展新产品的研发。

\section{2 数控车削凸轮轴的工艺设计}

\section{1 凸轮轴型面的车削准备}

(1) 机床的选择。采用宝鸡机床 CK7520 数控车床搭建加工平台。

（2）车削刀具的选择。高速钢刀具容易磨得锋利的刃口, 并且有较好的韧性, 能承受较大的冲击, 比 较适用于凸轮的车削。

（3）刀具角度的选择。凸轮的两侧都有避空, 车刀主偏角选用 $75^{\circ}$, 这样刀尖角 $\varepsilon_{r}$ 较大, 能够较大的 切削力, 并有利于切削散热。车削加工在凸轮加工中属粗加工, 因毛坏材料选用 45 号钢, 前角与后角按 中碳钢选择参考值, 前角选用 $25^{\circ} \sim 30^{\circ}$, 后角选用 $6^{\circ} \sim 8^{\circ}$, 副后角可通过作图确定。

(4) 走刀方式选择。可用的切削进刀方式有径向进刀和轴向进刀，此处采用轴向进刀，主切削刃的切 削状况将得到改善, 磨损较大的角度仅仅是刀具的副后角, 前角与主后角在切削的过程中不会发生变化, 副切削刃参与切削的长度仅为每转进给的量, 刀具受力状况优于径向进刀,

切削用量的确定。切削用量含背吃刀量、切削速度、进给量等指标。不同的切削用量适用于不同的切 削方法。背吃刀量 $a_{p}$ 一般选 $4 \mathrm{~mm}$; 按照普通外圆车削 45 钢材料切削线速度一般在 $0.333^{\sim} 0.5 \mathrm{~m} / \mathrm{s}$ 之间, 也 就是 $20^{\sim} 30 \mathrm{~m} / \mathrm{min}$ 之间 ${ }^{[6]}$, 这里选用 $25 \mathrm{~m} / \mathrm{s}$, 凸轮最大直径大约 $50 \mathrm{~mm}$, 那么由:

$$
v=\pi d n
$$

可得:

$$
n=\frac{v}{\pi d}=\frac{25 \mathrm{~m} / \mathrm{min}}{3.14 \times 0.05 \mathrm{~m}} \approx 159 \mathrm{r} / \mathrm{min}
$$

可以取较小的转速值 $n=150 \mathrm{r} / \mathrm{min}$; 车削凸轮型面时, 为稳妥起见, 适当降低进给速度, 这里选择 $\mathrm{f}=0.3 \mathrm{~mm} / \mathrm{r}$ 。那么分钟进给速度为:

\section{$0.3 \mathrm{~mm} / \mathrm{r} \times 150 \mathrm{r} / \mathrm{min}=45 \mathrm{~mm} / \mathrm{min}$}

\section{3 基于 Matlab 的切削用量优化}

\section{1 建立优化数学模型}

(1) 已知条件。车削加工 45 钢材料的凸轮轴, 背吃刀量 $a_{p}=4 \mathrm{~mm}$, 车刀刀尖圆弧半径 $\mathrm{r}_{\mathrm{c}}=0.5 \mathrm{~mm}$, 调质处 理后材料硬度 HB200。由优化设计数学模型, 求最优经济加工成本 $\mathrm{C}_{\min }$ 条件下的进给量 $f *$ 与切削速度 $\sim *$ 。 
查机械手册 ${ }^{[7]}$ 得常数 $\mathrm{a}=1.25, \mathrm{~b}=0.87, \mathrm{k}=212$, 最大切削线速度为 $\mathrm{V}_{\max }=30 \mathrm{~m} / \mathrm{min}$, 最小切削线速度为 $\mathrm{V}_{\min }=20 \mathrm{~m} / \mathrm{min}$ 。最大允许粗糙度 $\mathrm{R}_{\text {amax }}=0.08 \mathrm{~mm}$, 最大进给量 $\mathrm{f}_{\max }=0.45 \mathrm{~mm} / \mathrm{r}$, 最小进给量 $\mathrm{f}_{\min }=0.3 \mathrm{~mm} / \mathrm{r}$, 稳定切 削区限制值为 $q=2000$, 最大切削功率 $P_{\text {cmax }}=11 \mathrm{KW}$ 。

(2) 确定优化变量。车削中, 如果增加进给量 $f$ 和切削速度 $v$, 切削时间会减少, 但刀具寿命就会降 低。所以，加工成本最小化的优化问题就是探寻 $f$ 和 $v$ 的最佳值问题。因此，把进给量 $f$ 和切削速度 $v$ 作 为优化问题的设计变量, 该问题就是一个 $X=\left[x_{1}, x_{2}\right]^{T}=[v, f]^{T}$ 的二维变量优化问题 ${ }^{[8]}$ 。

（3）确定目标函数。由题目中所给已知条件，把单个凸轮轴零件加工成本 $\mathrm{C}$ 值作为该问题的优化目标 函数:

$$
f(X)=\mathrm{C}=437.5+28.798 /(f \cdot v)+0.6121566 \times f^{0.82} \cdot v^{3.35}
$$

把设计变量代入目标函数:

$$
f(X)=C=437.5+28.798 /\left(x_{2} \cdot x_{1}\right)+0.6121566 \times x_{2}{ }^{0.82} \cdot x_{1}^{3.35}
$$

(4) 确立约束条件。

性能约束:

(1)最大车削功率 $\mathrm{P}_{\mathrm{cmax}}$ 的限制条件: $P_{c}=F_{e v} / 1000 \leq \mathrm{P}_{\mathrm{cmax}}$;

(2)最大切削力限制条件: $F_{c}=9.8 \mathrm{k} f^{\mathrm{a}} \mathrm{a}_{\mathrm{p}}^{\mathrm{b}} \leq \mathrm{F}_{\mathrm{cmax}}$;

(3)稳定切削区的限制条件： $v^{\mathrm{e}} f \geq \mathrm{q}$;

(4)最大理论表面粗粘度 $\mathrm{R}_{\text {max }}$ 的限制条件: $R=\frac{f^{2}}{8 \mathrm{r}_{\mathrm{c}}} \leq \mathrm{R}_{\text {max }}$ ； 边界约束:

(1) 进给量限制条件: $\mathrm{f}_{\text {min }} \leq f \leq \mathrm{f}_{\text {max }}$ ；

(2) 切削速度限制条件: $v_{\text {min }} \leq v \leq v_{\text {max }}$ ；

其中 $a 、 b 、 e 、 k 、 q$ 是常数, 可由机械手册中的切削规范查得。

\section{1 用 Matlab 优化工具箱求解数学模型}

综合利用前面的车削加工工艺参数优化设计数学模型和 $\mathrm{SQP}^{[9]}$ 方法, 应用 Matlab 优化工具箱函数 ${ }^{[10]}$, 因为这里属于不等式约束, 所以 $A$ eq $=b e q=C_{e q}=0$, 另外 : $1 b=[20,0,0.3] ; u b=[30,0,0.45] ; A=[1,0,0,1] ; b=[30,0,0,0.45]$ 。

Matlab 优化工具箱里面的 fmincon 函数要用到两个 M 函数，分别定义如下：

(1) function $\mathrm{f}=$ CuttingFunct $(x): f=437.5+\frac{28.798}{x(1) \times x(2)}+0.6121566 \times x(1)^{3.35} \times x(2)^{0.82}$

(2) function $\left[C, C_{e q}\right]=$ CuttingConstr $(x)$ 。

$$
[x, \text { fval }]=\text { fmincon }\left(\text { 'CuttingFunct' }, \mathrm{x}_{0}, \mathrm{~A}, \mathrm{~b},[],[], \mathrm{lb}, \mathrm{ub},{ }^{\prime} \text { CuttingConstr', options }\right)
$$


运算结果: $\left[x_{1}, x_{2}\right]=[23.5589,0.2339]$

$$
\text { fval }=6.4702 \times 10^{3}
$$

可以看出当切削速度 $v$ 取 $23.5589 \mathrm{~m} / \mathrm{s}$, 进给量取 $\mathrm{f}=0.2339 \mathrm{~mm} / \mathrm{r}$ 时, 成本最优值为 $6.4702 \times 10^{3}$ 。从 结果可以看出, 应用 Matlab 软件的优化工具箱对目标问题进行优化, 容易获得比传统方法更好的结果, 特别是在批量生产时，综合考虑加工成本和加工效率，对企业的经济效益更具意义。

\section{4 结论}

本文结合凸轮轴的传统车削加工工艺，在凸轮型面数控车削的基础上，大胆提出了合并车削工艺，缩 短凸轮轴加工工艺链的方案, 并对车削工艺过程的具体问题, 诸如机床平台选择问题、刀具角度选择问题、 走刀方式选择问题等进行了探讨, 尤其是对切削用量的选择问题用 Matlab 软件的优化工具箱做了详尽具 体的分析, 结果证明此优化方法是可行的。

\section{5 致谢}

作者非常感谢国家自然科学基金资助项目 (No. 51605145); 河南省教育厅自然科学研究项目（No. 12 B520019 ); 河南科技大学高级别项目培育基金（No. 2011CX016）给予的支持。

\section{参考文献:}

[1] 杨建明. 凸轮轴数控加工工艺研究 [J]. 中国制造业信息化，2012，41 (17)：71-73.

[2] 夏焕金，陆忠民. 凸轮轴凸轮型面车铣加工工艺的研究 [J]. 机车车辆工艺，2010，(5)：11-13.

[3] 郑孟昆. 高速高精凸轮轴磨床砂轮架优化设计[D]. 武汉：华中科技大学，2012.

[4] 机车柴油机凸轮轴型线设计与工艺优化[D]. 大连: 大连理工大学, 2007.

[5] 陈宏均．机械加工技师综合手册 [M]．北京：机械工业出版社，2007.

[6] 张以鹏. 实用切削手册 [M]. 沈阳：辽宁科学技术出版社，2007.

[7] 闻邦椿等．机械设计手册 [M]．第五版．北京：机械工业出版社，2008.

[8] 何兵，车林仙，刘初升，等. 车削加工工艺参数优化 [J]。现代制造工程，2013，(2)：79-83.

[9] 杜晋. MATLAB 在车削加工优化设计中的应用 [J]。煤矿机械, 2008, 29(9)：103-104.

[10］曾怀灵，贺建国．MATLAB 在机械优化设计中的应用 [J]．采矿技术，2012，12(3)：88-91.

\section{Acknowledgements}

The authors gratefully acknowledge the National Nature Science Foundation (Project No. 51605145), Henan Provincial Department of Education Natural Science Research Project (Project No. 12B520019), and the Major and previous pre-research project of Henan University of Science and Technology (Project No. 2011CX016)

\section{References:}

[1] Yang Jianming. Research on NC Processing Technology of Camshaft [J]. Manufacturing Information Engineering Of China, 2012, 41(17): 71-73.

[2] Xia Huanjin, Lu Zhongmin. Study on Turning and Milling Processing Technology of Camshaft Cam Surfaces [J]. Locomotive \& Rolling Stock Technology, 2010, (5): 11-13.

[3] Zheng Mengkun. Optimization Design of High Speed and High Precision Camshaft Grinding Wheel Frame [D]. Wuhan: Huazhong University of Science and Technology, 2012.

[4] Profile Design and Process Optimization for Camshaft of Locomotive Diesel Engine [D]. Dalian: Dalian University of Technology, 2007 
[5] Chen Hongjun. Comprehensive Handbook of Machining Technicians [M]. Beijing: China Machine Press, 2007

[6] Zhang Yipeng. Practical Cutting Handbook [M]. Shenyang: Liaoning Science and Technology Press House, 2007.

[7] Wen Bangchun, etc. Mechanical Design Manual (The Fifth Edition) [M]. Beijing: China Machine Press, 2008

[8] He Bing, Che Linxian, Liu Chusheng, etc. Optimization of Turning Process Parameters [J]. Modern Manufacturing Engineering, 2013, (2): 79-83.

[9] Du Jin. Application of MATLAB in Optimization Design of Turning Process [J]. Coal Mine Machinery, 2008, (9): 103-104.

[10]Zeng Huailing, He Jianguo. Application of MATLAB in Mechanical Optimization Design [J]. Mining Technology, 2012, 12 (3): 88-91. 